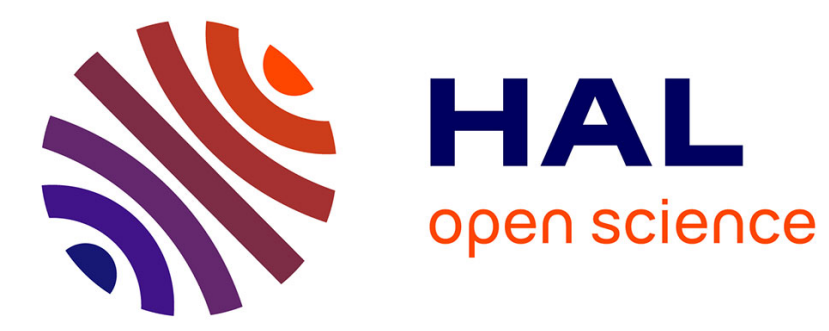

\title{
On the Effects of an Antireflection Coating Impairment on the Sensitivity to Optical Feedback of AR/HR Semiconductor DFB Lasers
}

Frédéric Grillot

\section{- To cite this version:}

Frédéric Grillot. On the Effects of an Antireflection Coating Impairment on the Sensitivity to Optical Feedback of AR/HR Semiconductor DFB Lasers. IEEE Journal of Quantum Electronics, 2009, 45 (6), pp.720-729. 10.1109/JQE.2009.2013155 . hal-00501879

\section{HAL Id: hal-00501879 \\ https://hal.science/hal-00501879}

Submitted on 12 Jul 2010

HAL is a multi-disciplinary open access archive for the deposit and dissemination of scientific research documents, whether they are published or not. The documents may come from teaching and research institutions in France or abroad, or from public or private research centers.
L'archive ouverte pluridisciplinaire HAL, est destinée au dépôt et à la diffusion de documents scientifiques de niveau recherche, publiés ou non, émanant des établissements d'enseignement et de recherche français ou étrangers, des laboratoires publics ou privés. 


\title{
On the Effects of an Antireflection Coating Impairment on the Sensitivity to Optical Feedback of AR/HR Semiconductor DFB Lasers
}

\author{
Frédéric Grillot, Member, IEEE
}

\begin{abstract}
The sensitivity to optical feedback of $1.55-\mu \mathrm{m}$ antireflection (AR)/high-reflection (HR) DFB semiconductor lasers is presented in this paper. The onset of the coherence collapse, which is the most critical feedback regime for optical transmissions, is theoretically investigated with a stress on its dependence with facet phase effects (FPEs). Taking into account FPEs on both facets, the sensitivity to optical feedback is evaluated with respect to both the coupling strength coefficient and the feedback level. The first part of the paper shows that due to the HR-facet, a distribution up to several decibels on the coherence collapse thresholds is predicted over the whole DFB laser population. The second part concentrates on the coherence collapse dependence with respect to the AR coating. Calculations show an enhancement of the coherent collapse threshold distribution up to $10 \mathrm{~dB}$ due to the AR coating impairment. These simulations are of first importance for optical transmissions since they show that for AR coatings beyond $10^{-4}$, the sensitivity to optical feedback of AR/HR DFB lasers is extremely difficult to evaluate from one laser to another. On the other hand, for AR coatings below $10^{-4}$, all feedback performances are directly connected to the laser wavelength, and DFB lasers can be easily selected for high bit rate isolator-free transmission.
\end{abstract}

Index Terms-Coherence collapse, DFB, external optical feedback, facet phase effects (FPEs).

\section{INTRODUCTION}

$\mathbf{T}$ HE extension of today's optical networks to the home requires the development of extremely low-cost laser sources [1]. While wafer fabrication techniques allow massive production, packaging remains a cost bottleneck, as it is not supported by parallel processing. Cost reduction must therefore be based on packaging simplification, such as flip-chip bonding and direct coupling of the laser into the fiber [2]. However, in order to realize an optical module without an optical isolator, the design of feedback resistant lasers continues to remain a challenge. It is well known that the performance of a semiconductor laser is strongly altered by any type of external optical feedback. Five distinct regimes based on spectral observation have been reported for $1.55-\mu \mathrm{m}$ semiconductor DFB [3]. The

Manuscript received July 07, 2007; revised June 06, 2008 and July 14, 2008. Current version published May 20, 2009.

The author is with the Center for High Technology Materials, The University of New Mexico, Albuquerque, NM 87106 USA, and also with the National Institute for Applied Sciences (INSA), Rennes 35043, France (e-mail: fgrillot@chtm.unm.edu).

Color versions of one or more of the figures in this paper are available online at http://ieeexplore.ieee.org.

Digital Object Identifier 10.1109/JQE.2009.2013155 laser sensitivity can be such that even under a feedback level in the percent range, the laser becomes unstable and starts operating within the so-called coherence collapse regime [4]. The main consequence of such a regime on the semiconductor laser is a drastic enhancement of the laser linewidth up to several gigahertz, which is very detrimental to most applications. In the important case of optical transmission, the coherence collapse leads to a strong degradation in the bit error rate (BER) when the laser is used as a transmitter, as theoretically [5] and experimentally [6] demonstrated. More generally, the prediction of the coherence collapse threshold remains an important feature for all applications requiring either a low noise level or a proper control of the laser coherence. Based on a weak coherent feedback hypothesis, the determination of the critical feedback threshold was analytically derived for Fabry-Perot lasers in [7]. An analytical method was also proposed to determine the feedback sensitivity of DFB lasers [8]. Both approaches concluded on the importance of calculating the coupling strength coefficient [9] corresponding to the strength of the coupling from a laser facet to an external cavity. Following [8], the coherence collapse threshold of DFB lasers having an antireflection (AR) coating on the emitting facet and a high-reflection (HR) coating on the rear facet has been calculated and compared to experimental results [10]. It was both theoretically and experimentally shown in [10] that, due to the HR-facet, the feedback sensitivity as well as the coherence collapse threshold exhibits a quasi-parabolic distribution due to facet phase effects (FPEs). The large dispersion among the critical levels observed for a given set of DFB lasers leads to a wide range of behavior under external optical feedback, which is detrimental to most applications. The goal of this paper is to investigate the coherence collapse dependence with respect to the AR coating. We show that small variations on the order of $10^{-3}$ of the AR coating can critically affect the laser dependence with respect to optical feedback.

The paper is organized as follows. In Section II, the theory of DFB lasers operating under external optical feedback is presented. At first, the generalized Lang and Kobayashi rate equations already reported in [11] and [12] are reintroduced. An expression of the laser pulsation variation serving for the calculation of both the feedback sensitivity and the coherence collapse threshold is derived from the generalized dynamical equations. General expressions of the coupling strength coefficients valid for any laser cavity come as a consequence of these equations. Numerical calculations are then conducted and the feedback sensitivity of DFB lasers is evaluated. Instead of assuming 
a facet phase distribution on the HR-facet only, facet phases on the AR-facet due to a residual reflectivity are also taken into account. Simulations reporting variations of both the coupling strength coefficient and the onset of the coherence collapse are presented and discussed in Section III. Finally, we summarize our results and conclusions in Section IV. These simulations are of first importance for optical transmissions since they show that for AR coatings beyond $10^{-4}$, the sensitivity to optical feedback of AR/HR DFB lasers is extremely difficult to evaluate from one laser to another. On the other hand, for AR coatings below $10^{-4}$, all feedback performances are directly connected to the laser wavelength, and DFB lasers can be selected for high bit rate isolator-free transmission.

\section{Generalized Rate Equations Under External OPTICAL FEEDBACK}

In this section, main results from [12] that deal with the generalization of the Lang and Kobayashi rate equations in the presence of external optical feedback are reintroduced to the reader. Thus, it is shown that an expression of the laser pulsation variation serving for the calculation of both the feedback sensitivity and the coherence collapse threshold is derived from these dynamical equations. As a consequence, general expressions of the coupling strength coefficients from a laser facet to an external cavity come as a consequence of these equations and are used to evaluate the feedback sensitivity of AR/HR DFB lasers with an AR coating impairment.

\section{A. Generalization of the Rate Equations Including External Optical Feedback}

In what follows, it is assumed that the cavity medium is isotropic and the laser perfectly index guided. In addition to these conditions, the longitudinal axis is only explicitly taken into account. Both transverse and lateral variations are accounted for by the effective dielectric constant. Longitudinal variations along the laser axis, which are related to the effects of the spatial hole burning (SHB), are included [13]. Based on the Green's functions theory and starting from the wave equation for the electromagnetic field [11], [14]-[16], it has been shown that the resolution of the generalized dynamical rate equations in the presence of external optical feedback can be written as follows [12]:

$$
\begin{aligned}
\frac{d P}{d t}= & \frac{2}{L} \int_{(L)} W_{N_{i}} \Delta N d z P+2 \operatorname{Im}\left[W_{p} \gamma\left(1-\rho_{k}^{2}\right) \frac{\xi_{0}(t-\tau)}{\xi_{0}(t)}\right] \\
& +F_{p}(t) \\
\frac{d \phi}{d t}= & \omega_{0}-\omega-\frac{1}{L} \int_{(L)} W_{N_{r}} \Delta N d z \\
& -\operatorname{Re}\left[W_{p} \gamma\left(1-\rho_{k}^{2}\right) \frac{\xi_{0}(t-\tau)}{P \xi_{0}(t)}\right]+F_{\phi}(t) \\
W_{N_{r}}= & \operatorname{Re}\left[\frac{\partial W / \partial N}{\partial W / \partial \omega}\right] \\
W_{N_{i}}= & \operatorname{Im}\left[\frac{\partial W / \partial N}{\partial W / \partial \omega}\right] \\
W_{p}= & \frac{\partial W / \partial \rho_{k}}{\partial W / \partial \omega}
\end{aligned}
$$

with $z$ being the longitudinal coordinate, $P$ the photon number inside the cavity, and $\phi$ the phase of the electrical field. In (1) and (2), $\omega_{0} / 2 \pi$ is the free-running laser frequency, $\omega / 2 \pi$ is the lasing frequency in the presence of optical feedback, $\gamma$ is the amplitude reflectivity of the external optical feedback coming from a distant reflecting point and being assumed to be such as $\gamma \ll 1$, $\tau=2 L_{e} / c$ is the external round-trip time (with $L_{e}$ the external cavity length), $\xi_{0}(t)$ is the complex electrical field defined as $\xi_{0}(t)=\sqrt{P(t)} e^{j \phi(t)}, F_{p}(t)$ and $F_{\phi}(t)$ are the Langevin forces in the time domain, and $\Delta N$ is the carrier density deviation induced by the external optical feedback. As it has been previously mentioned, longitudinal variations such as those on internal optical power are taken into account through the integral terms over the cavity length $L$. On the other hand, it is important to stress that expressions of $W_{N r}, W_{N i}$, and $W_{p}$ given by (3), (4), and (5) are linked to the Wronskian $W\left(\omega, N(z), \rho_{k}\right)$ that comes as a consequence of the resolution of the wave equation for the electromagnetic field [12]. Basically, the Wronskian describes the lasing conditions under external optical feedback and depends on the lasing frequency $\omega / 2 \pi$, the carrier density $N(z)$ as well as the amplitude reflectivity $\rho_{k}$ of the $k$-facet (with $k=r$ for the right facet and $k=1$ for the left facet). It has been shown that the Wronskian can be expressed as [16], [17]

$$
\begin{aligned}
W\left(\omega, N(z), \rho_{k}^{e q}\right)=W & \left(\omega_{0}, N_{0}(z), \rho_{k}\right)+\frac{\partial W}{\partial \omega} \Delta \omega \\
& +\frac{1}{L} \int_{(L)} \frac{\partial W}{\partial N} \Delta N d z+\frac{\partial W}{\partial \rho_{k}} \Delta \rho_{k}
\end{aligned}
$$

with $\Delta \omega=\omega-\omega_{0}, \Delta N=N-N_{0}$, and $\Delta \rho_{k}=\rho_{k}^{e q}-\rho_{k}$ is the variation of the $k$-facet reflectivity induced by external optical feedback [8], [18]

$$
\Delta \rho_{k}=\left(1-\rho_{k}^{2}\right) \gamma e^{-j \omega \tau} .
$$

Equation (6) shows that when the laser is exposed to external optical feedback, the lasing frequency and the carrier density distribution deviate from their original values. The oscillation condition corresponds to a zero in the Wronskian term, which serves to determine the laser longitudinal mode. Such a condition can be written as follows:

$$
W\left(\omega_{0}, N_{0}(z), \rho_{k}\right)=0 .
$$

As the Wronskian is complex, both the lasing frequency $\omega_{0} / 2 \pi$ and the carrier density distribution at threshold $N_{0}$ are completely determined from (8). Finally, it is important to note that in (1) and (2), the parameter $W_{p}$ is also complex such as $W_{\mathrm{p}}=\left|W_{p}\right| e^{-j \varphi}$ with $\left|W_{p}\right|$ the module and $\varphi$ the argument. The system described by (1) and (2) constitutes a generalization of the well-known Lang and Kobayashi rate equations [18] used to study Fabry-Perot lasers operating in the presence of optical feedback. It can be applied to any type of semiconductor lasers. By using (1) and (2) as well as the dynamic evolution of the carrier density, it has been shown in [12] that the variation of the angular frequency induced by external optical feedback can be written by the following relation:

$$
\Delta \omega \tau_{i}=-2 C_{k} \gamma \sqrt{1+\alpha_{H}^{2}} \sin \left(\varphi+\arctan \left(\alpha_{H}\right)\right)
$$


with $\alpha_{H}$ the linewidth enhancement factor and $\tau_{i}$ the internal round-trip time. In (9), $C_{k}$ represents the coupling strength coefficient of the $k$-facet, which can be expressed as

$$
C_{k}=\frac{j \tau_{i}}{2}\left(1-\rho_{k}^{2}\right) W_{p}
$$

Equation (10) constitutes a general expression of the coupling strength coefficient that takes into account the coupling from the $k$-facet to an external cavity. The coupling strength coefficient serves to quantify the sensitivity to external optical feedback of both the threshold gain and frequency variations [8], [18] of a semiconductor laser as well as to determine its coherence collapse threshold. Due to the coupling strength coefficient, the onset of the coherence collapse regime (also named critical feedback level) occurring at a certain feedback level $\Gamma=\Gamma_{C}$ (where $\Gamma=|\gamma|^{2}=P_{r} / P_{e}$ the feedback fraction with $P_{e}$ and $P_{r}$ being the emitted and reflected powers, respectively) can be determined by using the well-known analytical relation [7]

$$
\Gamma_{C}=\frac{\omega_{r}^{4} \tau_{i}^{2}}{16\left|C_{k}\right|^{2}\left(1+\alpha_{H}^{2}\right) \omega_{d}^{2}}
$$

where $\omega_{r}$ is the relaxation pulsation whereas $\omega_{d}$ represents the damping pulsation. It is important to stress that such a relation holds under the assumption of $\Gamma_{C}<-30 \mathrm{~dB}$ (weak optical feedback), $\alpha_{H}>1$, and $\omega_{p} \tau \gg 1$ with $p=r, d$ standing either for relaxation or damping. In (11), the ratio $\omega_{d} / \omega_{r}^{2}$ is usually defined as the $K$-parameter. This parameter whose typical value ranges from 0.1 to $0.5 \mathrm{~ns}$ is often used in the characterization of high-speed lasers to describe the damping of the response [19], [20]. The coherence collapse is the common name given to describe the complicated irregular dynamics that occurs when the laser is operating above and not too close to threshold. A lot of papers describe the coherence collapse regime as coexisting chaotic attractors [21] whereas others explain it as an important source of noise [22], [23]. As it has been previously mentioned, this regime is associated with a drastic increase of the spectral linewidth that leads to a strong degradation in the BER. As a result, the prediction of the coherence collapse threshold remains an important feature for all applications requiring either a low noise level or a proper control of the laser coherence.

\section{B. Case of a Fabry-Perot Laser}

As an example, the Wronskian of a Fabry-Perot laser without SHB effects can be written by the following relation [17]:

$$
W=2 j \beta \rho_{r}\left(\rho_{r} \rho_{l} e^{-2 j \beta L}-1\right)
$$

with $\rho_{r} \rho_{l} e^{-2 j \beta L}-1$ being the oscillation condition and $\beta$ the complex propagation constant, which can be expressed as

$$
\beta=n k_{0}+j \frac{G-\alpha}{2}
$$

where $n$ is the refractive index, $G$ the optical gain, and $\alpha$ the internal loss, all in the active region. After calculating $\partial W / \partial \omega$ and $\partial W / \partial \rho_{k}$ (with $\rho_{k}$ the amplitude reflectivity of the $k$-facet), $W_{p}$ can be expressed by the following relation:

$$
W_{p}=j \frac{1}{\rho_{k} \tau_{i}} .
$$

Then, by injecting (14) into (10), the coupling strength coefficient for a Fabry-Perot laser can be written as

$$
C_{k}=\frac{1-R_{k}}{2 \sqrt{R_{k}}} \quad \forall k=r, 1
$$

with $R_{k}=\left|\rho_{k}\right|^{2}$ being the reflectivity in intensity of the $k$-facet. Equation (15) coincides with the well-known relation of the Fabry-Perot coupling strength coefficient published in [24]. Thus, by assuming an as-cleaved laser for which $R_{k}=0.32$, a typical value of 0.60 is found for both facets. Fabry-Perot lasers will not be investigated in the following since only spectral single-mode devices such as DFB lasers are considered.

\section{Case of a DFB Laser}

The Wronskian of a conventional DFB laser can be written as follows [8], [17]:

$$
W=4 \beta_{B} \frac{\sigma}{\kappa} \frac{F(\sigma L)}{\left(\widetilde{\rho}_{l}-\frac{\hat{X}}{j \kappa}\right)\left(\widetilde{\rho}_{r}-\frac{X}{j \kappa}\right)}
$$

where $\beta_{B}$ is the Bragg wavenumber, $L$ is the length of the laser cavity, and $\kappa$ is the grating coupling coefficient (e.g., internal feedback induced by the grating). In (16), $\widetilde{\rho}_{k}$ is the complex amplitude reflectivity on the $k$-facet whose expression is given by $\widetilde{\rho}_{k}=\varphi_{k} e^{j \varphi_{k}}$ where $\rho_{k}$ is the amplitude reflectivity of the $k$-facet and $\varphi_{k}$ is the facet phase that describes the position of the facet in the corrugation. $\sigma$ is the complex propagation constant such as

$$
\sigma^{2}=\kappa^{2}+q^{2}
$$

where $q=\alpha_{0}-j \delta_{0}$ with $\alpha_{0}$ and $\delta_{0}$, respectively, being the laser losses and the Bragg deviation, both without optical feedback. In (16), the terms $X$ and $\hat{X}$ are defined by $X=\sigma+q$ and $\hat{X}=-\sigma+q$.

Let us note that when an AR coating is used on both facets, DFB lasers that have a uniform grating emit on two longitudinal modes that are symmetrically located with respect to the Bragg wavelength. These two longitudinal modes have the same losses and define the stopband of the laser. In order to obtain a single-mode laser, an HR coating can be, for instance, applied on the rear facet to break the longitudinal symmetry. However, due to the HR coating, interference effects between the grating and the facets make the lasing properties highly dependent on cleavage plane variations as small as a part of a wavelength. As a consequence, the lasing wavelength of a DFB laser is extremely difficult to control and the laser does not emit systematically at the Bragg wavelength and can lase at another wavelength located within the laser stopband. Thus, in order to account for this 
random phenomenon, the so-called Bragg deviation $\delta_{0}$ (e.g., the detuning) has to be introduced as

$$
\delta_{0}=\beta-\beta_{\mathrm{B}}
$$

where $\beta$ is the lasing wavenumber.

Finally, let us note that the function $F(\sigma L)$ describing the threshold condition is given by the following relation:

$$
\begin{aligned}
F(\sigma L)=\left(1-\widetilde{\rho}_{l} \frac{\hat{X}}{j \kappa}\right) & \left(1-\widetilde{\rho}_{r} \frac{\hat{X}}{j \kappa}\right) \\
& -\left(\widetilde{\rho}_{l}-\frac{\hat{X}}{j \kappa}\right)\left(\widetilde{\rho}_{r}-\frac{\hat{X}}{j \kappa}\right) e^{2 \sigma L} .
\end{aligned}
$$

By using (10) and (16), the derivative functions used for the determination of $W_{p}$ can be written such as

$$
\begin{aligned}
& \frac{\partial W}{\partial \omega}=-\frac{j}{\sigma} q \frac{n}{c} \frac{\partial W}{\partial \sigma} \\
& \frac{\partial W}{\partial \sigma}=4 \beta_{B} \frac{\sigma}{\kappa} \frac{d F / d \sigma}{\left(\widetilde{\rho}_{r}-\frac{\hat{X}}{j \kappa}\right)\left(\widetilde{\rho}_{l}-\frac{\hat{X}}{j \kappa}\right)} \\
& \frac{\partial W}{\partial \widetilde{\rho}_{l}}=4 \beta_{B} \frac{\sigma}{\kappa} \frac{1}{\widetilde{\rho}_{r}-\frac{\hat{X}}{j \kappa}} \frac{d F / d \widetilde{\rho}_{l}-\frac{\hat{X}}{j \kappa}}{\widetilde{\rho}_{l}} \\
& \frac{\partial W}{\partial \widetilde{\rho}_{r}}=4 \beta_{B} \frac{\sigma}{\kappa} \frac{1}{\widetilde{\rho}_{l}-\frac{\hat{X}}{j \kappa}} \frac{d F / d \widetilde{\rho}_{r}}{\widetilde{\rho}_{r}-\frac{\hat{X}}{j \kappa}} .
\end{aligned}
$$

Then, by using the set of (10) and (18)-(22), the coupling strength coefficient of each facet can be expressed such as

$$
\begin{aligned}
C_{l}= & \frac{2\left(1-\left|\rho_{l}\right|^{2}\right) e^{-j \varphi_{l}}\left(q^{2}+\kappa^{2}\right) L^{2}}{j \kappa L\left(1+\widetilde{\rho}_{l}^{2}\right)-2 \widetilde{\rho}_{l} q L} \\
& \times\left[\frac{1}{2 q L-\sum_{k=r, l} \frac{\left(1-\widetilde{\rho}_{k}^{2}\right) \kappa L}{2 j q L \widetilde{\rho}_{k}+\kappa L\left(1+\widetilde{\rho}_{k}^{2}\right)}}\right] \\
C_{r}= & \frac{2\left(1-\left|\rho_{r}\right|^{2}\right) e^{-j \varphi_{r}}\left(q^{2}+\kappa^{2}\right) L^{2}}{j \kappa L\left(1+\widetilde{\rho}_{r}^{2}\right)-2 \widetilde{\rho}_{r} q L} \\
& \times\left[\frac{1}{2 q L-\sum_{k=r, l} \frac{\left(1-\widetilde{\rho}_{k}^{2}\right) \kappa L}{2 j q L \widetilde{\rho}_{k}+\kappa L\left(1+\widetilde{\rho}_{k}^{2}\right)}}\right] .
\end{aligned}
$$

These coefficients denote the coupling strength from the laser to an external cavity. It can be seen from these equations that coefficients $C_{k}$ (with $k=r, l$ ) are only linked to the intrinsic laser characteristics such as the optical losses, the normalized Bragg deviation, the grating coupling coefficient, and complex reflectivities. Equations (23) and (24) also show that the optical field is affected both by $\varphi_{l}$ and $\varphi_{r}$ since the situation $\rho_{r} \neq 0$ and $\rho_{l} \neq 0$ is assumed. As a result, these generalized equations can be used to evaluate the influence of FPE occurring on the rear facet but also those induced on the front facet.

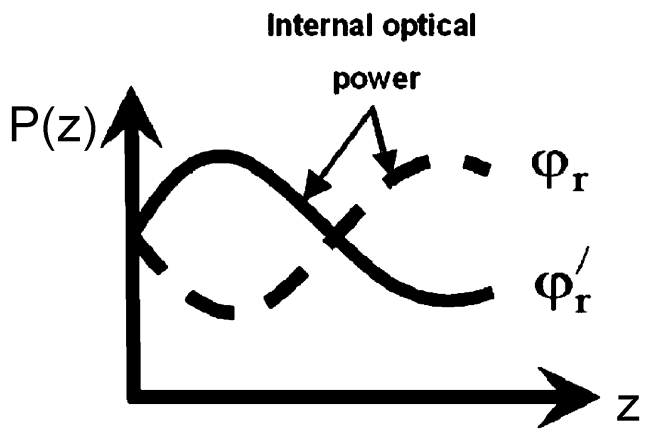

Fig. 1. Schematic illustration of the internal optical power redistribution due to changing reflectivity phases. The emitting power $\mathrm{P}$ is represented as a function of $\mathrm{z}$ corresponding to the longitudinal axis of the laser cavity.

In the case of a perfect AR coating such as $\rho_{r}=0$ or $\rho_{l}=0$, (23) and (24) are simply reduced to

$$
\begin{aligned}
C_{r} & =\frac{-j\left[(q L)^{2}+(\kappa L)^{2}\right]}{q L\left[\kappa L\left(1+\widetilde{\rho}_{r}^{2}\right)-j \widetilde{\rho}_{r}\right]+2 j \widetilde{\rho}_{r}(q L)^{2}-\kappa L} e^{j \varphi_{r}} \\
C_{l} & =\frac{\left[(q L)^{2}+(\kappa L)^{2}\right]\left[2 \widetilde{\rho}_{r}(q L) / \kappa L-j\left(1+\widetilde{\rho}_{r}^{2}\right)\right]}{q L\left[\kappa L\left(1+\widetilde{\rho}_{r}^{2}\right)-j \widetilde{\rho}_{r}\right]+2 j \widetilde{\rho}_{r}(q L)^{2}-\kappa L} e^{j \varphi_{l}} .
\end{aligned}
$$

Those expressions that hold only if a perfect AR coating (e.g., $\left.\rho_{k}=0\right)$ is assumed are similar to those already published in [8]. Let us emphasize that the study of FPE in DFB lasers is extremely important since FPE leads to a modification of the phase matching between the grating and the facet. For instance, depending on the facet phase at the HR-facet (assuming FPE negligible on the front facet), the repartition of the internal optical power $P$ is modified and varies along the longitudinal axis (z) of the laser cavity. Thus, as shown in Fig. 1, between a facet case $\varphi_{r}$ and another $\varphi_{r}^{\prime}$, the repartition of the field within the laser cavity is changed. As a result, due to the power redistribution, the coupling strength coefficient and the sensitivity to optical feedback through the critical feedback level are altered.

In what follows, the coupling strength coefficients as well as the coherence collapse thresholds are calculated on $1.55-\mu \mathrm{m}$ AR/HR DFB lasers. As an example, FPEs are taken into account on both facets by assuming an AR coating ranging from 0 to $10^{-3}$ and a $95 \%$ rear reflectivity. Although the front facet is AR-coated, FPE can have a significant effect. In the following, it is shown that extremely small AR coatings, below $10^{-3}$, are required in order to counteract the AR FPE deleterious phenomena.

\section{Numerical RESUlts AND DiscusSiON}

\section{A. Position of the Problem}

Let us consider an AR/HR DFB laser as the one shown in Fig. 2. In order to optimize the laser external efficiency, a high 95\% HR coating is assumed on the rear facet while the front facet AR coating is kept as small as possible. In Fig. 2, an external optical feedback $\gamma$ produced on the AR-coated side and induced by a reflector of amplitude reflectivity $R$ is assumed. The amplitude reflectivity of the AR/HR DFB laser is denoted 


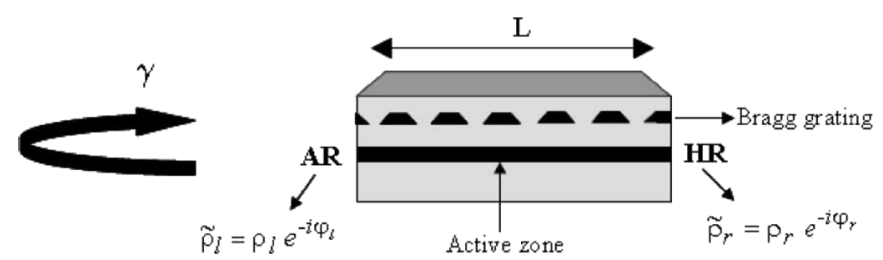

Fig. 2. Design of the AR/HR straight section studied laser. The amount $\gamma$ denotes the optical feedback reinjected into the cavity through the left facet. $\widetilde{\rho}_{1}$ and $\widetilde{\rho}_{\mathrm{r}}$ correspond to the complex amplitude reflectivities on the AR- and HR-facet, respectively, while $L$ represents the length of the laser cavity.

as $\rho_{r}$ and $\rho_{l}$ for the high-reflectivity- and antireflective-coated facets, respectively. Since the front facet is AR-coated, FPE can be at first assumed negligible on the front facet. However, it will be demonstrated in the following that this assumption is not true when AR coatings in the order of $10^{-4}$ are reached. By considering $\left|\rho_{r}\right|^{2}=0.95$ and $0 \leq\left|\rho_{l}\right|^{2} \leq 10^{-3}$, the modulus of the complex coefficient $C_{l}$ related to the facet submitted to external optical feedback is calculated via (23) for each phase case $\varphi_{k}$ with $0 \leq \varphi_{k} \leq 2 \pi(k=r$ and $k=l)$. Then, the sensitivity to optical feedback is evaluated by calculating the so-called coherence collapse threshold for a given output power. Through (11), it is important to stress that the coherence collapse threshold depends on FPE via the complex coefficient $C_{l}$ and the resonance frequency $\omega_{r}$ whose expression is given by the relation $\omega_{r}=A \sqrt{P / \eta\left(\varphi_{r}\right)}$ where $A, P$, and $\eta\left(\varphi_{r}\right)$ are, respectively, a constant coefficient, the output power, and the external efficiency that depends on facet phases [20], [25]. As a result, the current is varied to counteract the facet phase dependence of the external efficiency $\eta\left(\varphi_{r}\right)$ so as to keep the output power constant in the simulations. Finally, let us note that the lasers parameters used for the calculations are equal to $\tau_{i}=7.5 \mathrm{ps}, \alpha_{H}=2.0$, $L=300 \mu \mathrm{m}, \omega_{d}=12 \mathrm{GHz}$, and $A=2 \mathrm{GHz} /(\mathrm{mA})^{1 / 2}$ for the internal round-trip time, the linewidth enhancement factor, the laser cavity length, the damping frequency, and the $A$-parameter (modulation efficiency), respectively. The normalized grating coupling coefficient $\kappa L$ chosen for the simulations equals either 0.3 or 1.0. All these parameters correspond to typical DFB laser data close to those used in [10]. In the following, both the coupling strength coefficient and the coherence collapse threshold are calculated. More particularly, the effects of an AR coating impairment on the sensitivity to external optical feedback of semiconductor DFB lasers are discussed.

\section{B. Numerical Results and Discussion}

In Fig. 3, simulations showing the variations of both the coupling strength coefficient and the coherence collapse threshold versus the normalized Bragg deviation $\delta L$ are depicted for a DFB laser. In the calculations, reflectivities are, respectively, $\left|\rho_{l}\right|^{2} \equiv 0$ and $\left|\rho_{r}\right|^{2}=0.95$. In this situation, FPE occurs only on the rear facet since a zero AR reflectivity is assumed. Thus, quasi-parabolic distributions exhibiting either a maximum or a minimum located at the Bragg wavelength $(\delta L=0)$ are predicted. The best feedback resistant laser is obtained for a laser emitting in the middle of the stopband. This situation corresponds to the highest coherence collapse threshold $(\sim-47 \mathrm{~dB})$ [or to the lowest coupling strength coefficient $(\sim 6)]$. On the other hand, the worst situation for the laser is predicted for $\varphi_{r}=$
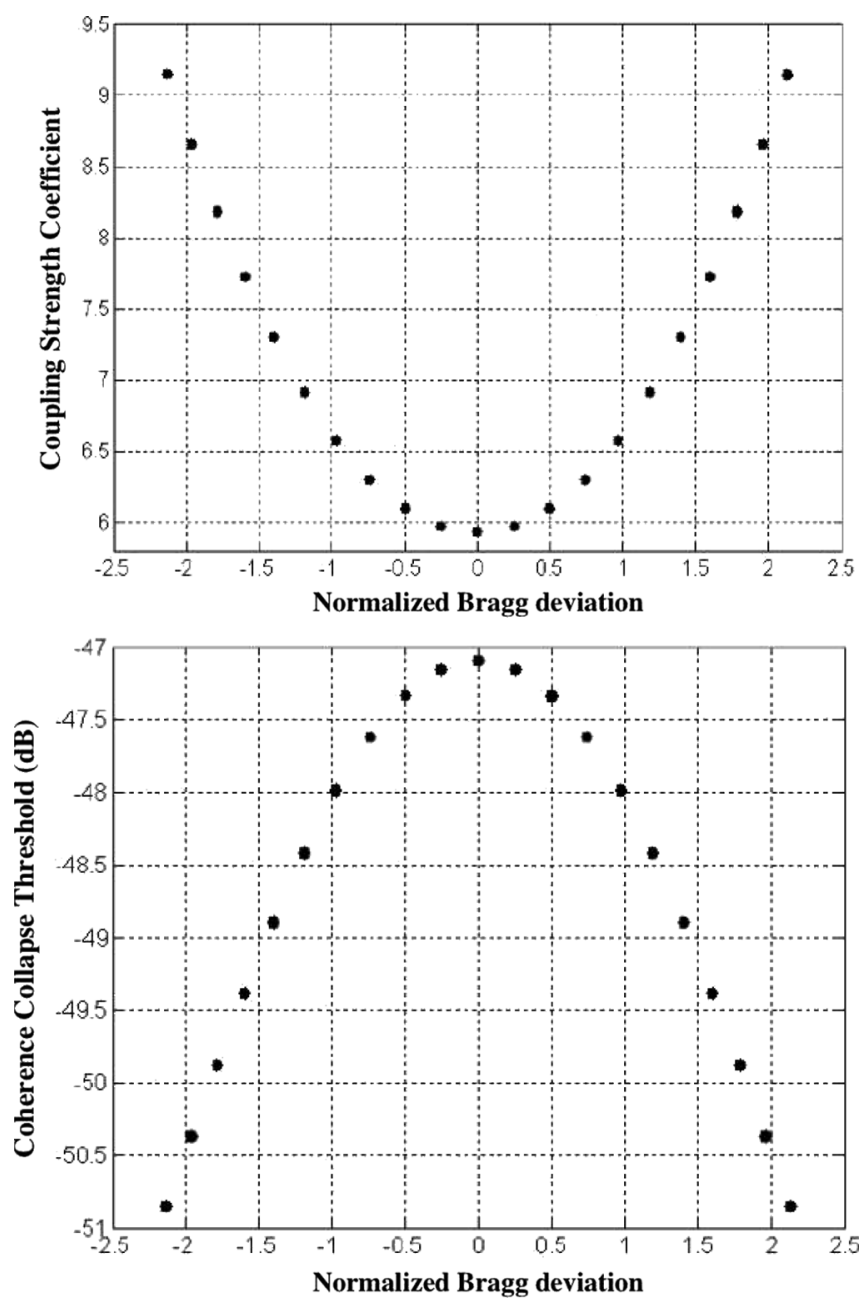

Fig. 3. Calculated coupling strength coefficients and calculated coherence collapse thresholds for a DFB laser with $\left|\rho_{1}\right|^{2} \equiv 0$ and $\left|\rho_{r}\right|^{2}=0.95$. The normalized grating coupling coefficient is $\kappa L=0.3$.

$2 \pi$ and for $\varphi_{r}=0$. In that case, the laser has two degenerate modes on both sides of the stopband. For a normalized Bragg deviation in the range from $\delta L=-2$ to $\delta L=+2$, the overall variation of the calculated coherence collapse threshold reach up to $\sim 4 \mathrm{~dB}$. Hence, a dependence of the coherence collapse threshold with FPE is theoretically predicted. Let us note that such results have already been confirmed experimentally [10]. Thus, due to the HR coating, the coherence collapse threshold is linked to FPE. This property is of first importance and can be useful to select the best feedback resistant device over a whole DFB laser population just by measuring the Bragg deviation via an optical spectrum analyzer (OSA).

Let us now assume a DFB laser with an AR reflectivity not strictly equal to zero: FPE now occurs on both facets. Keeping the laser parameters unchanged, variations of the calculated coupling strength coefficient and the coherence collapse threshold have been recalculated with respect to $\varphi_{l}$. Their dependence as a function of the normalized Bragg deviation is depicted in Fig. 4 for a DFB laser with $\kappa L=0.3$ as well as $\left|\rho_{l}\right|^{2}=10^{-6}$ and $\left|\rho_{r}\right|^{2}=0.95$. The parabolic distribution previously demonstrated is still present since the facet phase conditions do not change on the HR-facet. As the 

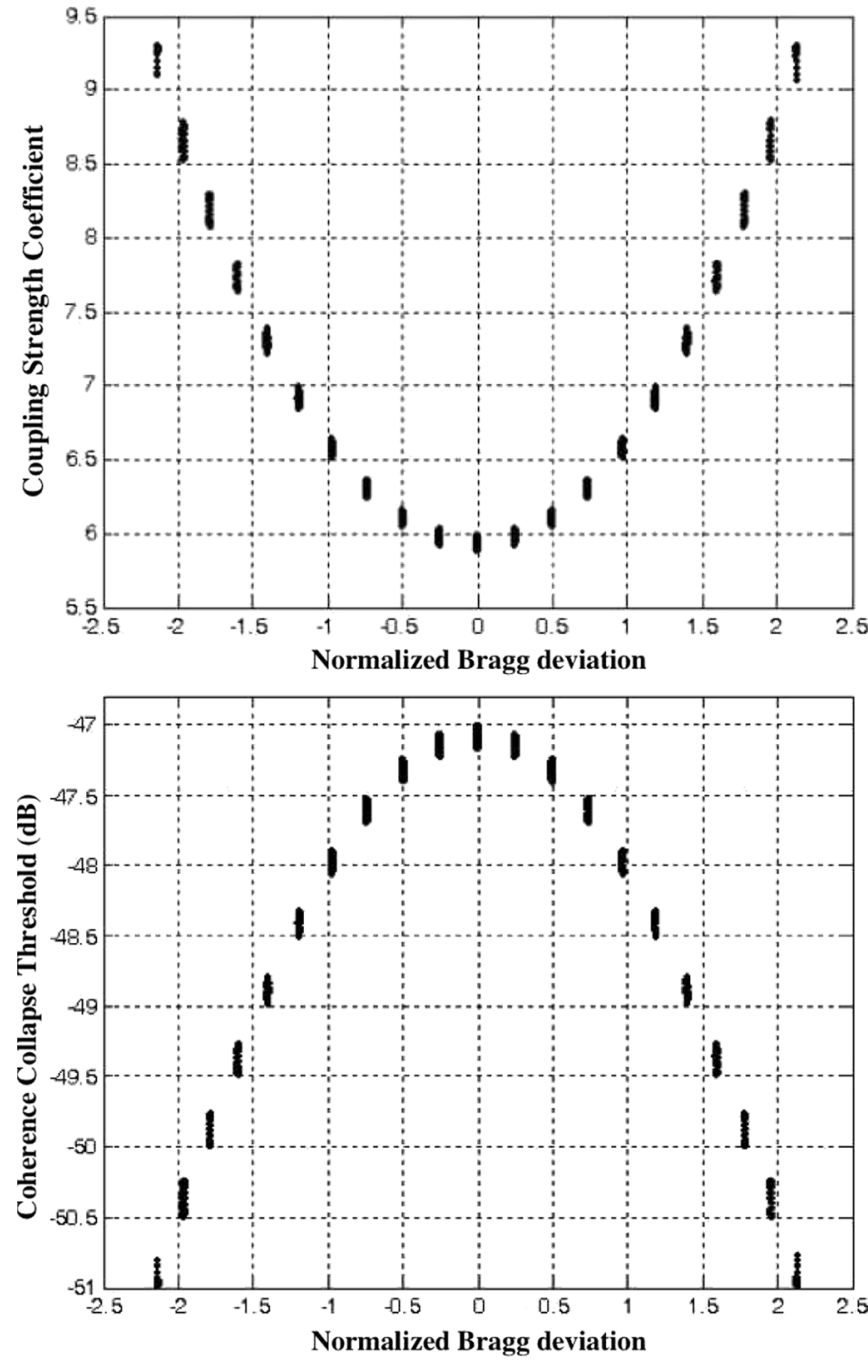

Fig. 4. Calculated coupling strength coefficient and calculated coherence collapse threshold for a DFB laser with $\left|\rho_{l}\right|^{2}=10^{-6}$ and $\left|\rho_{r}\right|^{2}=0.95$. The normalized grating coupling coefficient is $\kappa L=0.3$.

reflectivity of the front facet does not exceed $10^{-6}$, only a slight perturbation, which remains negligible, is predicted on the calculated coherence collapse thresholds. However, when increasing the reflectivity of the AR-facet to $\left|\rho_{l}\right|^{2}=10^{-5}$, as shown in Fig. 5, quasi-ellipses arise around the main parabolic distribution. Thus, for a given Bragg deviation, the variation on the critical feedback level that was negligible for $\left|\rho_{l}\right|^{2}=10^{-6}$ is now about $\sim 1 \mathrm{~dB}$ for $\left|\rho_{l}\right|^{2}=10^{-5}$. Despite the small effect observed at these levels of AR impairments, the results show that all along the first parabolic distribution, the amplitudes of several ellipses enhance both with the Bragg deviation and with the AR reflectivity. It is worth noting that the influence of FPE due to the feedback from the front facet of an integrated DFB laser has already been reported in [26].

Fig. 6 reports the same calculations as previously but for $\left|\rho_{l}\right|^{2}=2 \times 10^{-4}$. The same behavior is simulated but now it can be seen that FPEs are considerably modified. The amplitude of each ellipse is drastically enhanced and this phenomenon increases with the normalized Bragg deviation. For a laser emitting in the middle of the stopband, the highest resistance
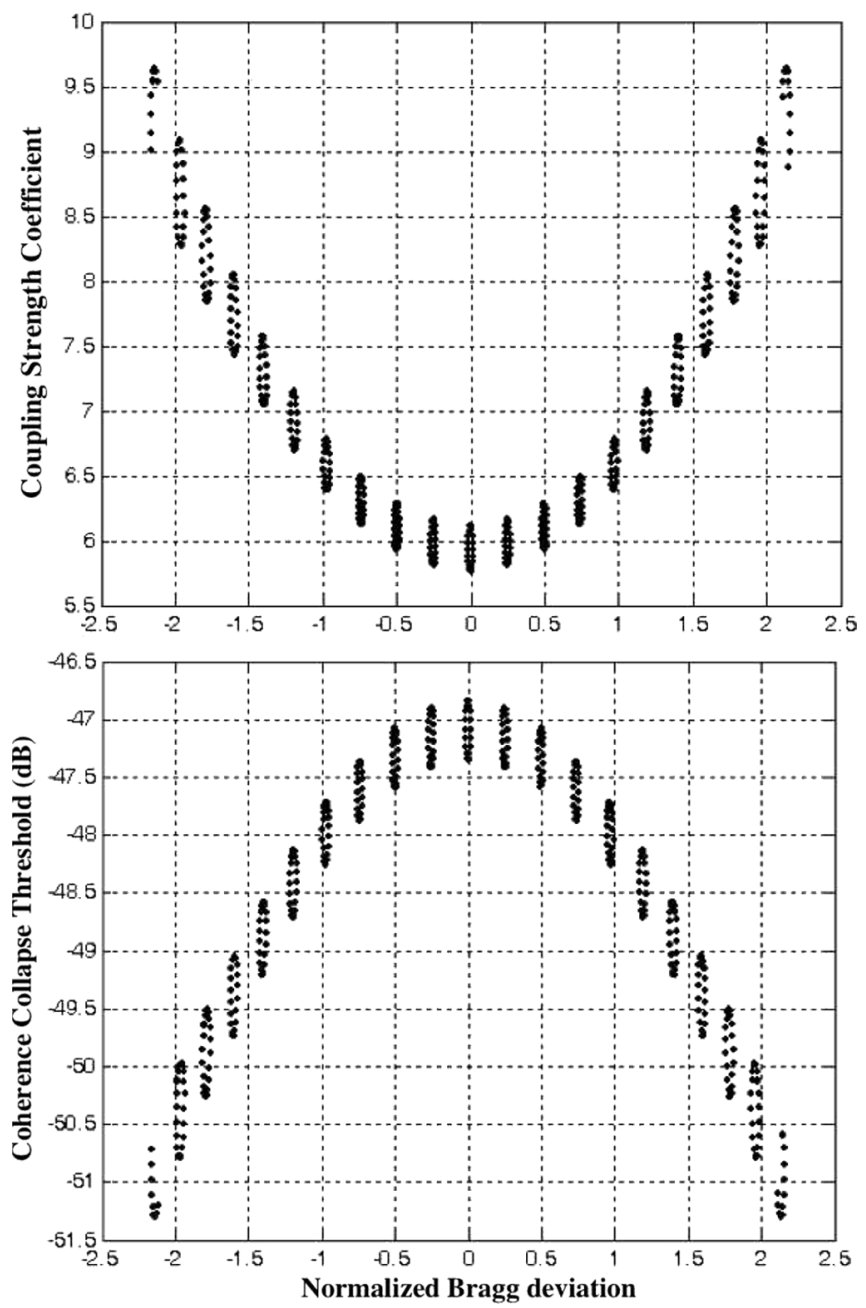

Fig. 5. Calculated coupling strength coefficient and calculated coherence collapse threshold for a DFB laser with $\left|\rho_{l}\right|^{2}=10^{-5}$ and $\left|\rho_{r}\right|^{2}=0.95$. The normalized grating coupling coefficient is $\kappa L=0.3$.

to optical feedback is still obtained with a coherence collapse threshold of $\sim-47 \mathrm{~dB}$. However, by varying $\varphi_{l}$ between 0 and $2 \pi$, FPE due to the AR-facet induces a supplementary variation close to $\sim 2.5 \mathrm{~dB}$. If the laser is significantly detuned from the Bragg wavelength, the sensitivity to optical feedback is more pronounced. For instance, on the edge of the parabolic distribution, HR-facet cases that are either $\varphi_{r}=0$ or $\varphi_{r}=2 \pi$ lead to the lowest coherence collapse threshold $(\sim-51 \mathrm{~dB})$. Keeping $\varphi_{r}=0$ or $\varphi_{r}=2 \pi$ and varying $0 \leq \varphi_{l} \leq 2 \pi$ induces a stronger dispersion around $-51 \mathrm{~dB}$ that can go up to $\sim 4 \mathrm{~dB}$. Larger variations are expected up to $10 \mathrm{~dB}$ if the accuracy of the AR is worse (e.g., $\left|\rho_{1}\right|^{2}>2 \times 10^{-4}$ ). An example is given in Fig. 7 where calculations are done for $\left|\rho_{l}\right|^{2}=10^{-3}$. Stronger variations on the coherence collapse thresholds up to $\sim 5 \mathrm{~dB}$ can be observed for a laser emitting in the middle of the stopband $(\delta L=0)$ while they reach $\sim 10 \mathrm{~dB}$ on the stopband edges $(\delta L= \pm 3)$. Additionally, simulations show that a significant overlap arises among the different ellipses. This overlap seems to be strengthened when DFB lasers emitting on the stopband edges are considered. Indeed, it has been previously demonstrated that when a slight AR impairment is considered, all ellipses that are located around the main parabolic distribution remain quite well 

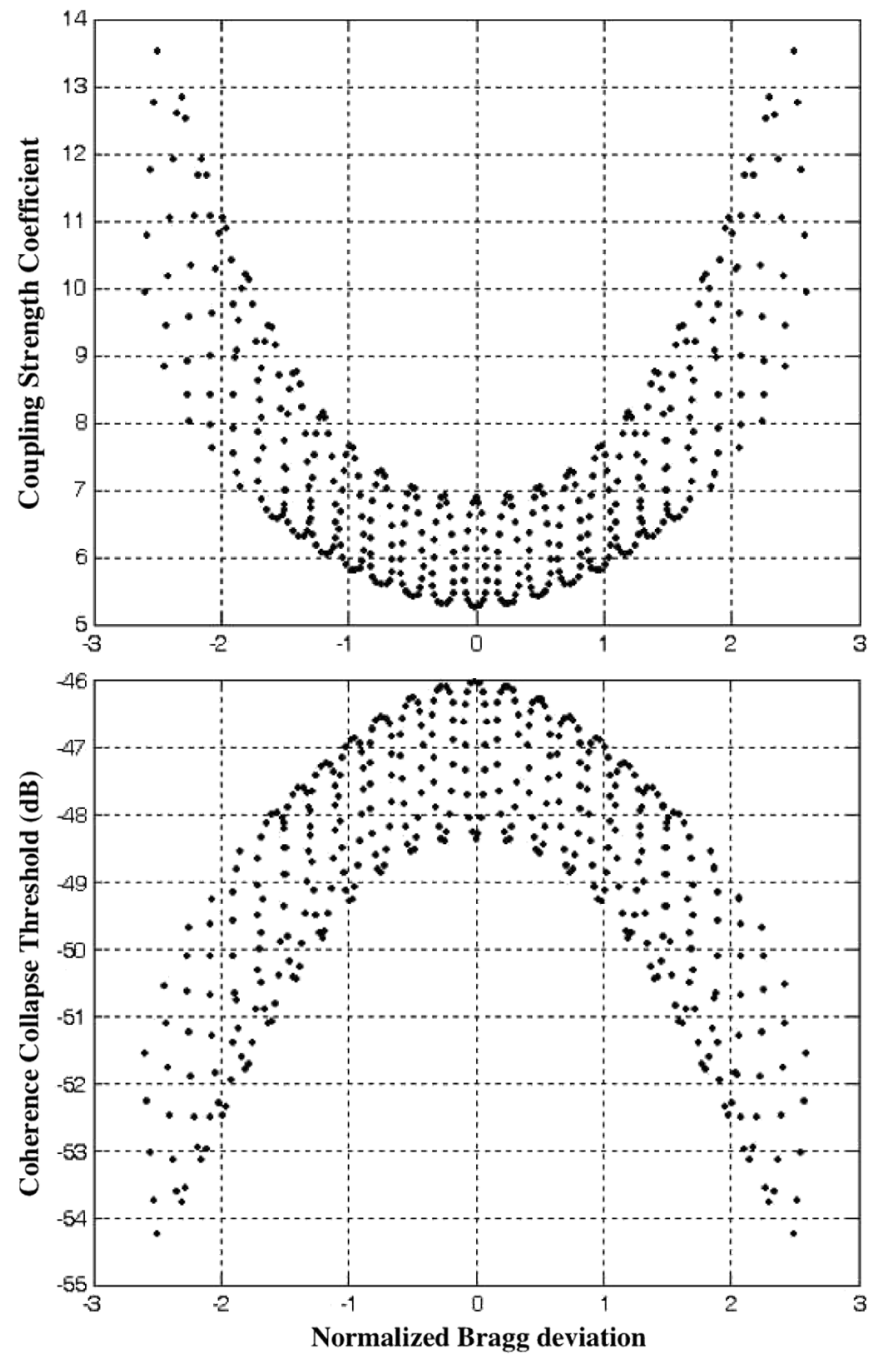

Fig. 6. Calculated coupling strength coefficient and calculated coherence collapse threshold for a DFB laser with $\left|\rho_{1}\right|^{2}=2 \times 10^{-4}$ and $\left|\rho_{r}\right|^{2}=0.95$. The normalized grating coupling coefficient is $\kappa L=0.3$.

separated from each other. In the case of a stronger AR coating impairment, such a situation does not appear clearly: all ellipses cross each other along the main parabolic distribution, breaking the original symmetry of the coherence collapse distribution. This situation is now very different than the one observed in the previous cases because the selection of the best-feedback resistant lasers cannot be performed by measuring the laser detuning. Indeed, all rules of selections are now much more complicated since a laser emitting at the Bragg wavelength can be more sensitive to optical feedback that one emitting on the stopband edge. Fig. 8 shows the distribution of the coherence collapse thresholds in the $\left(\varphi_{r}, \varphi_{l}\right)$ plane. For Fig. 8(a)-(c), the reflectivity of the front facet is, respectively, equal to $\left|\rho_{l}\right|^{2}=0,\left|\rho_{l}\right|^{2}=10^{-5}$, and $\left|\rho_{l}\right|^{2}=2 \times 10^{-4}$. As no FPE occurs in case Fig. 8(a), a symmetrical distribution is observed: for a given value of $\varphi_{r}$, the coherence collapse threshold remains constant for all values of $\varphi_{l}$. For instance, if $\varphi_{r}=\pi$, the coherence collapse threshold follows a vertical line when $\varphi_{l}$ increases from 0 to $2 \pi$ and remains equal to $\sim-47 \mathrm{~dB}$. This result is consistent with simulations exhibited in Fig. 3 where the lowest feedback sensitivity
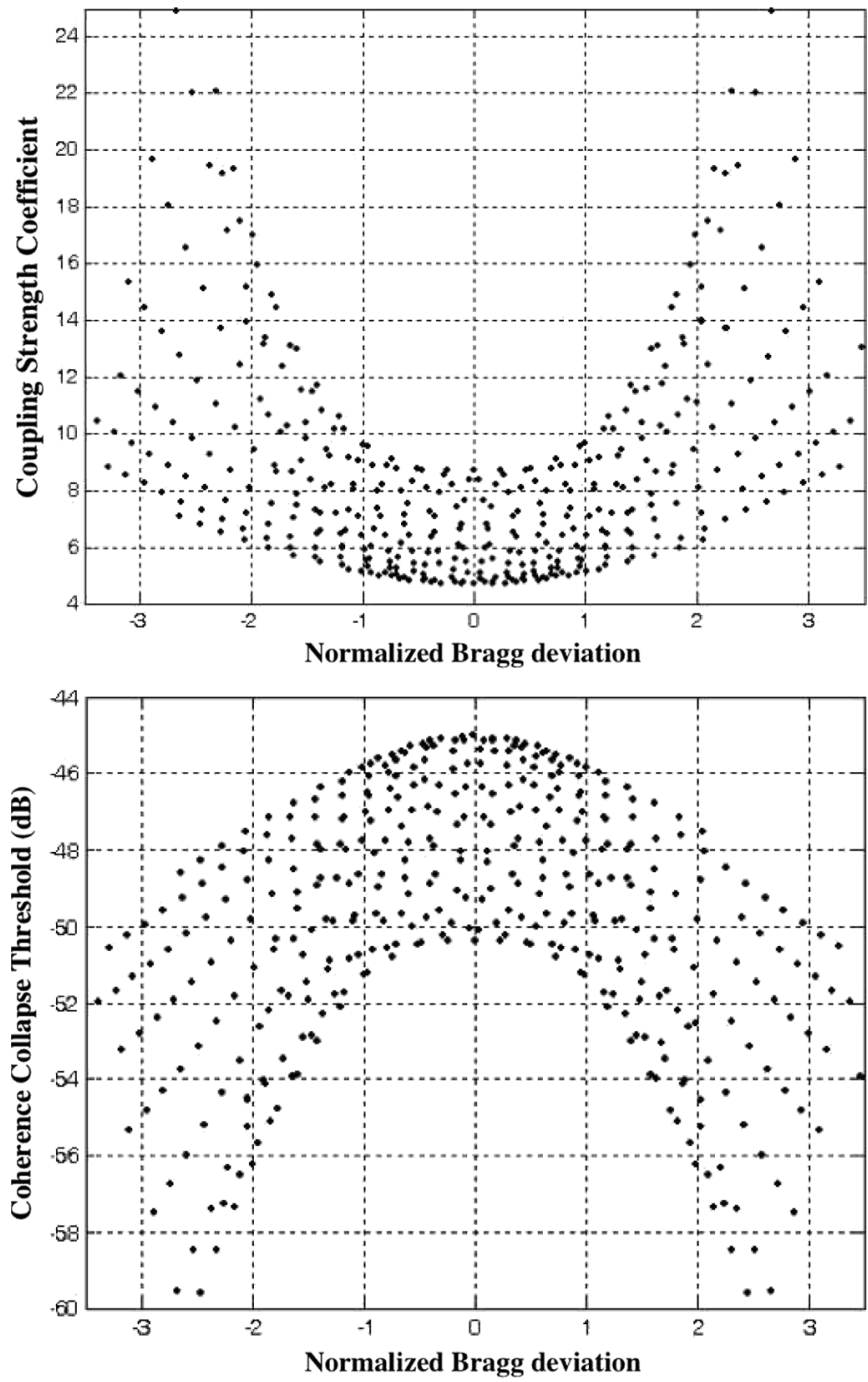

Fig. 7. Calculated coupling strength coefficient and calculated coherence collapse threshold for a DFB laser with $\left|\rho_{1}\right|^{2}=10^{-3}$ and $\left|\rho_{r}\right|^{2}=0.95$. The normalized grating coupling coefficient is $\kappa L=0.3$.

is obtained for $\varphi_{r}=\pi$. The situation is now quite different in Fig. 8(b) where some undulations start occurring in the distribution. This effect is correlated with the occurrence of the different ellipses that start arising around the parabolic distribution and that lead to some slight variations of the coherence collapse threshold. However, for DFB lasers emitting close to the Bragg wavelength, the coherence collapse threshold variation remains negligible as previously observed. As an example, for $\varphi_{r}=\pi$, the coherence collapse threshold does not change and stays close to $-47 \mathrm{~dB}$ for all values of $\varphi_{l}$. Finally, in Fig. 8(c), simulation shows that the sensitivity of the laser to optical feedback is strengthened. Indeed, for $\varphi_{r}=\pi$, the coherence collapse threshold is very $\varphi_{l}$-dependent and ranges from $\sim-49$ to $\sim-46 \mathrm{~dB}$. In that case, as the amplitudes of all ellipses increase with the value of the reflectivity of the front facet, the symmetry of the distribution is different than the one depicted in Fig. 8(a). For $\varphi_{r}=0$ or $\varphi_{r}=2 \pi$ and for $\varphi_{l}$ increasing from 0 to $2 \pi$, a good agreement with the results depicted in Fig. 6 is obtained 
(a)

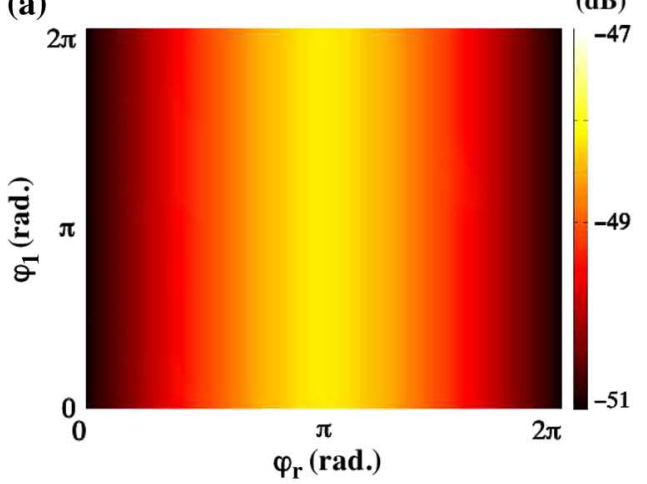

(b)

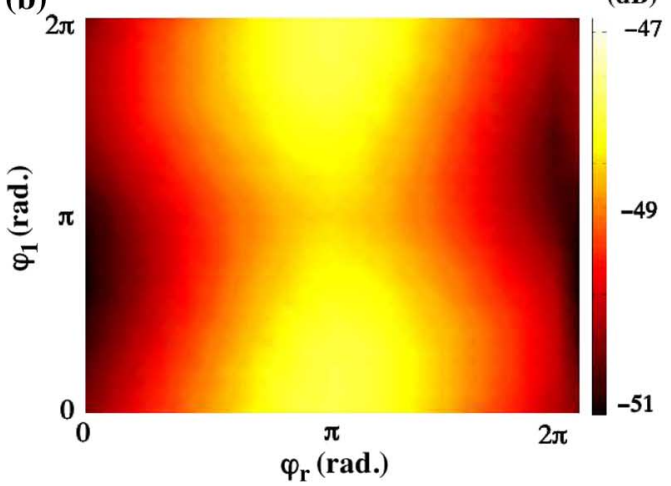

(c)

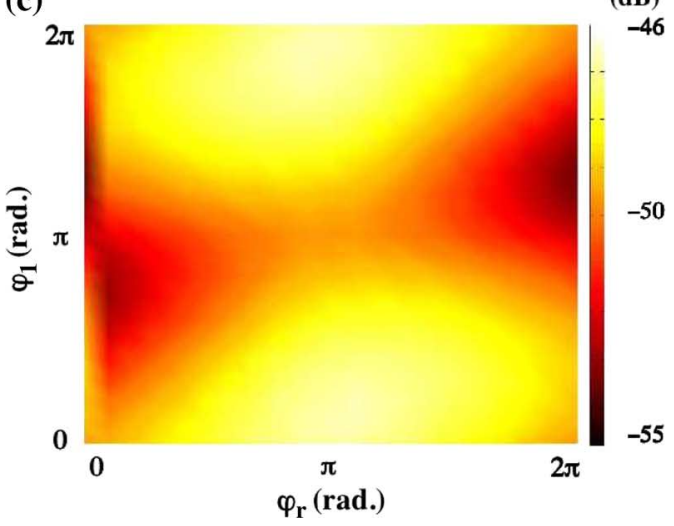

Fig. 8. Distributions of the coherence collapse thresholds (in decibels) in the $\left(\varphi_{r}, \varphi_{l}\right)$ plane calculated for $\left|\rho_{r}\right|^{2}=0.95, \kappa L=0.3$. (a) $\left|\rho_{l}\right|^{2}=0$. (b) $\left|\rho_{l}\right|^{2}=10^{-5}$. (c) $\left|\rho_{l}\right|^{2}=2 \times 10^{-4}$.

since the calculated coherence collapse thresholds range from $\sim-55$ to $\sim-50 \mathrm{~dB}$ on the stopband edges.

Thus, simulations show that the investigation of FPE on the coupling strength coefficient as well as on the coherence collapse threshold is an important feature in the case of AR/HR DFB lasers. Instead of assuming only facet phases on the rear facet, complementary facet phases occurring on the front facet and due to a residual reflectivity have also been taken into account in the calculations. In all cases, an expected result is obtained: the better the AR coating, the smaller the amplitude of the ellipse. This last point is determinant because the degradation of the penalty in optical transmissions occurs within the coherence collapse regime [6], [27]. Thus, the higher the onset of the critical feedback level, the better the BER measurements (a)

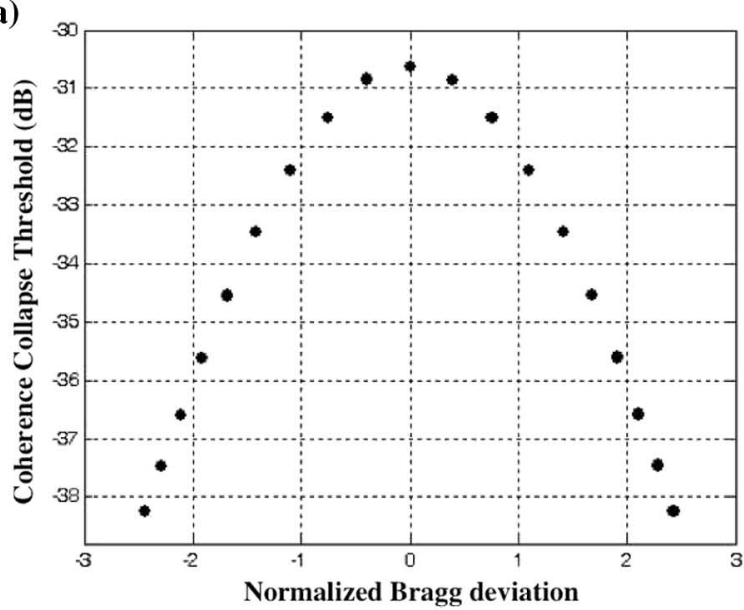

(b)

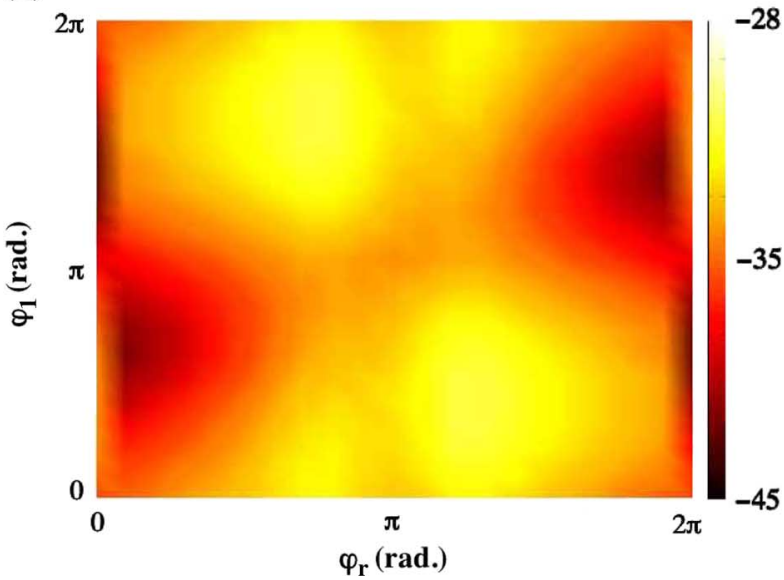

Fig. 9. Case of an AR/HR DFB laser with $\left|\rho_{r}\right|^{2}=0.95$ and $\kappa L=1.0$. (a) Coherence collapse thresholds calculated for $\left|\rho_{l}\right|^{2}=0$. (b) Distributions of the coherence collapse thresholds (in decibels) in the $\left(\varphi_{r}, \varphi_{l}\right)$ plane calculated for $\left|\rho_{l}\right|^{2}=5 \times 10^{-3}$

and the better the behavior of the laser in transmission. Simulations show that FPEs provoke a significant feedback dispersion in terms of coherence collapse threshold. As a result, these numerical calculations can be a strong input for telecommunication applications because they show that a high degree of accuracy on the AR coating is required to avoid supplementary FPE. In all cases, extremely small AR coatings, below $10^{-4}$, are required in order to counteract the AR FPE deleterious phenomenon, which is necessary to improve the quality of optical transmissions. For AR coatings on the order of $10^{-5}$, all feedback performances can be connected to the laser wavelength whereas for AR coatings beyond $10^{-4}$, simulations show that the sensitivity to optical feedback of AR/HR DFB lasers is extremely difficult to evaluate. It is somewhat important to stress that FPE linked to the residual reflectivity remains lower than those induced by the HR-facet. A better situation can be obtained by assuming the case of a DFB laser with a higher grating coupling coefficient. Indeed, it is well known that the resistance to optical feedback can be improved by using a DFB laser with a higher grating coupling coefficient. For instance, in Fig. 9(a), the calculated coherence collapse threshold variation is depicted versus the normalized Bragg deviation for $\left|\rho_{r}\right|^{2}=0.95,\left|\rho_{l}\right|^{2}=0$, 
and $\kappa L=1.0$. Calculations show that a critical feedback level as high as $\sim-31 \mathrm{~dB}$ is predicted for a DFB laser emitting in the middle of the stopband (compared to $\sim-47 \mathrm{~dB}$ predicted for $\kappa L=0.3$ ). When considering DFB lasers emitting on the stopband edge, coherence collapse thresholds do not exceed $-39 \mathrm{~dB}$ (compared to $\sim-51 \mathrm{~dB}$ predicted for the case of $\kappa L=0.3$ ). Fig. 9(b) shows the distribution of the coherence collapse thresholds in the $\left(\varphi_{r}, \varphi_{l}\right)$ plane for $\kappa L=1.0$ and $\left|\rho_{l}\right|^{2}=5 \times 10^{-3}$. Despite a stronger AR coating impairment, calculations show a lower asymmetry in the distribution compared to the one exhibited in Fig. 8(c) for $\kappa L=0.3$ and $\left|\rho_{l}\right|^{2}=10^{-3}$. As a result, the sensitivity to optical feedback of AR/HR DFB lasers can be improved by increasing the normalized grating coupling coefficient. Thus, a better homogeneity can be obtained under such conditions since stronger AR coating impairments have to be considered to observe significant variations on the coherence collapse thresholds. In any case, let us note that in order to avoid any FPE, the best situation can be reached when single-mode DFB lasers using tapered section and coated AR on both facets are designed. In that case, it has been demonstrated that no FPE occurs and a remarkable feedback uniformity is obtained among feedback performances [28].

\section{CONCLUSION}

This paper reports a numerical study on the effects of facet phases on the sensitivity to optical feedback of AR/HR DFB lasers. It has been shown that these single-mode components used for telecom applications suffer from FPE. Thus, due to FPE occurring on the rear facet coated HR, it has been demonstrated that the coupling strength coefficients as well as the coherence collapse thresholds are linked to the normalized Bragg deviation through a quasi-parabolic distribution. Then, taking into account an AR coating impairment, it has been shown that FPE arising on the front facet induces a second distribution located across the first parabolic one. This second dispersion, which is composed of several ellipses, enhances the sensitivity to optical feedback. In all cases, simulations have shown that extremely small AR coatings, below $10^{-4}$, are required in order to counteract the AR FPE deleterious phenomenon, which is necessary to improve the quality of optical transmissions. But above all, these numerical calculations demonstrate that for AR coatings on the order of $10^{-5}$, all feedback performances can be easily connected only to one parameter such as the laser wavelength. As a result, all lasers emitting at wavelengths matching favorable facet phases can be selected for high bit rate isolatorfree transmissions according to the recommended International Telecommunication Union (ITU) return loss specifications. On the other hand, it has been demonstrated that for AR coatings beyond $10^{-4}$, the sensitivity to optical feedback is much more difficult to evaluate from a laser to another. The laser wavelength cannot be used as a selection criterion since it is hard to say if a laser emitting in the middle of the stopband is really better than one emitting on the edge.

\section{REFERENCES}

[1] Y. Tohmori, Y. Itaya, and H. Toba, " $1.3 \mu \mathrm{m}$ spot-size converter integrated laser diodes (SS-LDs) for access network applications," in Proc. LEOS 1997, pp. 270-271, Paper TuQ1, unpublished.

[2] B. Fernier, K. Adams, C. Artigue, T. Barrou, A. Göth, E. Grard, W. Jörg, D. Keller, J. L. Lafragette, A. Lestra, P. Pagnod, S. Rabaron, J. M. Rainsant, J. Scherb, D. Toullier, D. Tregoat, and W. Rehm, "1.3 $\mu \mathrm{m}$ low cost plastic module for $622 \mathrm{Mbit} / \mathrm{s}$ transmission at $85^{\circ} \mathrm{C}$," in Proc. ECOC 1998, vol. 1, pp. 445-446.

[3] R. W. Tkach and A. R. Chraplyvy, "Regimes of feedback effects in 1.5 $\mu \mathrm{m}$ distributed feedback lasers," J. Lightw. Technol., vol. LT-4, no. 11, pp. 1655-1661, Nov. 1986.

[4] D. Lenstra, B. H. Verbeek, and A. J. Den Boef, "Coherence collapse in single-mode semiconductor lasers due to optical feedback," IEEE J. Quantum Electron., vol. QE-21, no. 6, pp. 674-679, Jun. 1985.

[5] R. B. Clarke, "The effect of reflections on the system performances of intensity modulated laser diodes," J. Lightw. Technol., vol. 9, no. 6, pp. 741-749, Jun. 1991.

[6] F. Grillot, B. Thedrez, F. Mallecot, C. Chaumont, S. Hubert, M. F. Martineau, A. Pinquier, and L. Roux, " $2.5 \mathrm{Gbit} / \mathrm{s}$ transmission characteristics of $1.3 \mu \mathrm{m}$ DFB lasers with external optical feedback," IEEE Photon. Technol. Lett., vol. 14, no. 1, pp. 101-103, Jan. 2002.

[7] J. Helms and K. Petermann, "A simple analytic expression for the stable operation range of laser diodes with optical feedback," IEEE J. Quantum Electron., vol. 26, no. 5, pp. 833-836, May 1990.

[8] F. Favre, "Theoretical analysis of external optical feedback on DFB semiconductor laser," IEEE J. Quantum Electron., vol. QE-23, no. 1, pp. 81-88, Jan. 1987.

[9] O. Nilsson and J. Buus, "Linewidth and feedback sensitivity of semiconductor diode lasers," IEEE J. Quantum Electron., vol. 26, no. 12, pp. 2039-2042, Dec. 1990.

[10] F. Grillot, B. Thedrez, O. Gauthier-Lafaye, M. F. Martineau, V. Voiriot, J. L. Lafragette, J. L. Gentner, and L. Silvestre, "Coherence collapse threshold of $1.3 \mu \mathrm{m}$ semiconductor DFB lasers," IEEE Photon. Technol. Lett., vol. 15, no. 1, pp. 9-11, Jan. 2003.

[11] C. H. Henry, "Theory of spontaneous emission noise in open resonators and its application to lasers and optical amplifiers," J. Lightw. Technol., vol. LT-4, no. 3, pp. 288-297, Mar. 1986.

[12] F. Grillot, G. H. Duan, and B. Thedrez, "Feedback sensitivity and coherence collapse threshold of semiconductor DFB lasers with complex structures," IEEE J. Quantum Electron., vol. 40, no. 3, pp. 231-240, Mar. 2004.

[13] W. S. Rabinovich and B. J. Feldman, "Spatial hole burning effects in distributed feedback lasers," IEEE J. Quantum Electron., vol. 25, no. 1, pp. 20-29, Jan. 1989.

[14] K. Ujihara, "Phase noise in a laser with output coupling," IEEE $J$. Quantum Electron., vol. QE-20, no. 7, pp. 814-818, Jul. 1984.

[15] G. H. Duan, P. Gallion, and G. Debarge, "Analysis of the phase-amplitude coupling factor and spectral linewidth of distributed feedback and composite-cavity semiconductor lasers," IEEE J. Quantum Electron., vol. 26, no. 1, pp. 32-43, Jan. 1990.

[16] B. Tromborg, H. Olesen, and X. Pan, "Theory of linewidth for multielectrode laser diodes with spatially distributed noise sources," IEEE J. Quantum Electron., vol. 27, no. 2, pp. 178-192, Feb. 1991.

[17] G. H. Duan, P. Gallion, and G. P. Agrawal, "Dynamic and noise properties of tunable multi-electrode semiconductor lasers including spatial hole burning and nonlinear gain," IEEE J. Quantum Electron., vol. 29, no. 3, pp. 844-855, Mar. 1993.

[18] R. Lang and K. Kobayashi, "External optical feedback effects on semiconductor injection laser properties," IEEE J. Quantum Electron., vol. QE-16, no. 3, pp. 347-355, Mar. 1980.

[19] K. Uomi, M. Aloki, T. Tsuchiya, and N. Chimone, "Dependence of relaxation oscillation frequency and damping $\mathrm{K}$ factor on the number of quantum wells in $1.55 \mu \mathrm{m}$ InGaAsP DFB lasers," IEEE Photon. Technol. Lett., vol. 3, no. 6, pp. 493-495, Jun. 1991.

[20] R. S. Tucker and I. P. Kaminow, "High frequency characteristics of directly modulated InGaAsP ridge waveguide and buried heterostructure lasers," J. Lightw. Technol., vol. LT-2, no. 4, pp. 385-393, Aug. 1984.

[21] J. Mork, B. Tromborg, and J. Mark, "Chaos in semiconductor lasers with optical feedback: Theory and experiment," IEEE J. Quantum Electron., vol. 28, no. 1, pp. 93-108, Jan. 1992. 
[22] J. Mork, B. Tromborg, and P. L. Christiansen, "Bistability and lowfrequency fluctuations in semiconductor lasers with optical feedback: A theoretical analysis," IEEE J. Quantum Electron., vol. 24, no. 2, pp. 123-133, Feb. 1988.

[23] B. Tromborg and J. Mork, "Non-linear injection locking dynamics and the onset of coherence collapse in external cavity lasers," IEEE $J$. Quantum Electron., vol. 26, no. 4, pp. 642-654, Apr. 1990.

[24] K. Petermann, "External optical feedback phenomena in semiconductor lasers," IEEE J. Sel. Topics Quantum Electron., vol. 1, no. 2, pp. 480-488, Jun. 1995.

[25] G. P. Agrawal and N. K. Dutta, Long-Wavelength Semiconductor Lasers. New York: Van Nostrand Reinhold, 1986.

[26] A. Lestra and P. Brosson, "Design rules for a low-chirp integrated DFB laser with an electroabsorption modulator," IEEE Photon. Technol. Lett., vol. 8, no. 8, pp. 998-1000, Aug. 1996.

[27] S. Azouigui, B. Dagens, F. Lelarge, J. G. Provost, A. Accard, F. Grillot, A. Martinez, Q. Zou, and A. Ramdane, "Tolerance to optical feedback of $10-\mathrm{Gb} / \mathrm{s}$ quantum-dash-based lasers emitting at $1.51 \mu \mathrm{m}$," IEEE Photon. Technol. Lett., vol. 19, no. 15, pp. 1181-1183, Aug. 2007.

[28] F. Grillot, B. Thedrez, F. Mallecot, C. Chaumont, S. Hubert, M. F. Martineau, and A. Pinquier, "Analysis, fabrication and characterization of $1.55 \mu \mathrm{m}$ selection-free tapered stripe DFB lasers," IEEE Photon. Technol. Lett., vol. 14, no. 8, pp. 1040-1042, Aug. 2002.

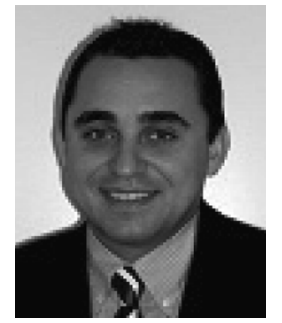

Frédéric Grillot (M'06) was born in Versailles, France, on August 22, 1974, 1974. He received the M.Sc. degree in physics from Dijon University, Dijon, France, in 1999 and the Ph.D. degree in electrical engineering from Besançon University, Besançon, France, in 2003.

His doctoral research activities were conducted within the Optical Component Research Department of Alcatel. Along with his doctoral work, he studied the effects of optical feedback in semiconductor lasers and the impact this phenomenon has on optical communications systems for hight-bit-rate transmissions. From May 2003 to August 2004, he was with the Institut d'Electronique Fondamentale, University of Paris-Sud, where he was focused on integrated optics modeling and Si-based passive devices for optical interconnects and telecommunications. In September 2004, he was appointed to the Institut National des Sciences Appliquées (INSA), Rennes, France, where he is currently an Associate Professor within the Materials and Nanotechnologies Department. His main research activities are focused on advanced laser diodes emitting at $1.55 \mu \mathrm{m}$ using new materials like quantum dots for low-cost applications. Since the beginning of 2008, he has also been a Visiting Research Professor of electrical and computer engineering with the University of New Mexico, Albuquerque, where he is leading research in optical science and optoelectrtonics at the Center for High Technology Materials.

Dr. Grillot is a member of the IEEE Photonics Society (formerly the IEEE Lasers and Electro-Optics Society) and La Société Française d'Optique. 